\title{
Effects of a human compact anti-ErbB2 antibody on gastric cancer
}

\author{
Carmine Fedele $\cdot$ Silvia Carvalho $\cdot$ Gennaro Riccio • \\ Rolando Paciello · Paolo Laccetti · Fernando Schmitt • \\ Claudia De Lorenzo
}

Received: 10 October 2012/ Accepted: 11 February 2013/Published online: 5 March 2013

(C) The International Gastric Cancer Association and The Japanese Gastric Cancer Association 2013

\begin{abstract}
Background Gastric cancer represents one of the most common causes of cancer deaths worldwide. Overexpression of ErbB2, a tyrosine kinase receptor involved in the pathogenesis of several human cancer types, has been reported also in gastric cancer. Thus, the inhibition of ErbB2 signal transduction pathways by the use of human antibodies could be a valuable strategy for the therapy of this type of cancer.

Methods We tested for the first time the antitumor effects on gastric cancer cells of Erb-hcAb, a novel fully human compact antibody, prepared in our laboratory, which
\end{abstract}

C. Fedele · G. Riccio - R. Paciello - P. Laccetti - C. De Lorenzo Dipartimento di Biologia Strutturale e Funzionale,

Università di Napoli Federico II, via Cinthia, 80126 Naples, Italy

S. Carvalho $\cdot$ F. Schmitt

Institute of Molecular Pathology and Immunology,

University of Porto, Porto, Portugal

S. Carvalho $\cdot$ F. Schmitt

Medical Faculty, University of Porto, Porto, Portugal

S. Carvalho

Department of Biological Regulation, Weizmann Institute

of Science, Rehovot, Israel

P. Laccetti

Dipartimento di Biologia, Università di Napoli Federico II,

Naples, Italy

C. De Lorenzo $(\square)$

Dipartimento di Medicina Molecolare e Biotecnologie Mediche,

Università di Napoli Federico II, Naples, Italy

e-mail: cladelor@unina.it

C. De Lorenzo

CEINGE Biotecnologie avanzate, via Gaetano Salvatore 486, 80145 Naples, Italy targets a different epitope of ErbB2 with respect to trastuzumab, the only anti-ErbB2 antibody currently in clinical use for both breast and gastric cancer therapy.

Results Herein we demonstrate that the in vitro and in vivo growth of gastric cancer cells is efficiently inhibited by Erb-hcAb, which shows antitumor effects on the NCIN87 cell line more potent than those observed for trastuzumab.

Conclusions Erb-hcAb could be a promising candidate in the immunotherapy of gastric cancer as it combines the antiproliferative effect associated with the inhibition of ErbB2 signaling on tumor target cells with the ability to induce antibody-dependent cellular cytotoxicity.

Keywords Immunotherapy - ErbB2/Her2 - Gastric cancer · Trastuzumab

\section{Introduction}

Gastric cancer is one of the most common human diseases and the second leading cause of tumor-related death worldwide [1]. Because the early disease is often asymptomatic, many gastric cancer patients are diagnosed when their cancer is already beyond curative surgery $[2,3]$.

Treatment options for these patients include radiotherapy and chemotherapy, but cancer progression is common, and the 5-year survival rate is less than $30 \%[2,3]$. Therefore, there is an urgent need to develop new highly potent therapies.

One possible therapeutic target is the ErbB2 oncoprotein, a member of the epidermal growth factor receptor (EGFR) family of tyrosine kinase receptors. Its overexpression occurs in many human cancer types and is implicated in malignant transformation and tumorigenesis 
$[4,5]$. ErbB2 is overexpressed in breast, ovary, non-small cell lung cancer, and in several other carcinomas [6-8], including gastric cancers, where it is associated with a poor clinical outcome $[9,10]$.

Trastuzumab is a humanized monoclonal antibody directed against the extracellular domain of the ErbB2 protein, currently the only one in clinical use for ErbB2targeted therapy either in the adjuvant setting or for the treatment of metastatic breast cancer [11, 12]. Many studies have shown a significant antitumor effect of trastuzumab in either in vitro or in vivo models of ErbB2positive gastric cancer [9, 10, 13-15]. Furthermore, when trastuzumab was given in combination with standard chemotherapy in a randomized phase III clinical trial (ToGA trial), it significantly improved overall survival [16]. These data indicate that trastuzumab is a valuable drug for the treatment of the ErbB2-positive subset of gastric cancers. Unfortunately, a large fraction of ErbB2-positive patients in the ToGA trial did progress on treatment $[16,17]$, thus suggesting that trastuzumab resistance might become a problem also in the therapy of gastric cancer, similar to what happened for breast cancer [18]. For this reason, several research strategies have focused on alternative ErbB2-directed treatments.

Recently, we produced a novel fully human anti-ErbB2 immunoagent [19], engineered by fusing erbicin, a human anti-ErbB2 single-chain antibody fragment (scFv) [20], with the Fc region of human IgG1. The human anti-ErbB2 antibody construct has been called Erb-hcAb for its 'compact' size $(100 \mathrm{kDa})$, compared with the full size $(155 \mathrm{kDa})$ of a natural IgG. It has been reported that Erb$\mathrm{hcAb}$ is capable of selectively binding to malignant cells that express ErbB2, and of inhibiting their growth in vitro and in vivo, with no effects on ErbB2-negative cells. Moreover, Erb-hcAb is endowed with both antibodydependent cellular cytotoxicity (ADCC) and complementdependent cytotoxicity (CDC) [19, 21].

More recently, it has been shown that Erb-hcAb does not display the cardiotoxic effect of trastuzumab in vitro on rat cardiomyocytes and in vivo on a mouse model, whereas trastuzumab was shown to be strongly toxic [22-25]. This difference was found to result from their different mechanism of action, which can explain their different effects: trastuzumab, in contrast to Erb-hcAb, induces apoptosis in cardiac cells [25] and inhibits the heterodimerization between ErbB2 and ErbB4 mediated by Neuregulin-1 [24]. Finally, Erb-hcAb is active in vitro and in vivo against some trastuzumab-resistant, ErbB2-positive breast cancer cell lines [26].

To validate Erb-hcAb as a possible additional or alternative treatment to trastuzumab, we analyzed its inhibitory effects on gastric tumor cells overexpressing ErbB2, which have been reported to be less sensitive to trastuzumab treatment. Here we prove for the first time the efficacy of Erb-hcAb on gastric cancer cells, and we show that it is endowed with an antitumor effect stronger than that of trastuzumab both in vitro and in vivo.

\section{Materials and methods}

\section{Cell lines}

The human cancer cell lines NCI-N87, MKN-7, MKN-45, AGS, SW480, SKBR3, MDA-MB-468, and MCF7 were purchased from the American Type Culture Collection (ATCC; Manassas, VA, USA) and were cultured in either RPMI (SKBR3, NCI-N87, MKN-7, MKN-45, AGS, SW480) or DMEM (MDA-MB-468 and MCF7) supplemented with $10 \%$ fetal calf serum and $1 \%$ penicillin/ streptomycin.

Cell lysis and immunoblotting analyses for detecting ErbB2 levels

Cells washed with phosphate-buffered saline (PBS) were collected by centrifugation at $1,200 \mathrm{rpm}$ for $7 \mathrm{~min}$. The pellet was resuspended in lysis buffer $(10 \mathrm{mM}$ Tris- $\mathrm{HCl}$, $\mathrm{pH} 7.4,0.5 \%$ Nonidet P-40, $150 \mathrm{mM} \mathrm{NaCl}$ ), containing complete proteases inhibitor (Boehringer Mannheim, Germany). After $20 \mathrm{~min}$ at $0{ }^{\circ} \mathrm{C}$, the extracts were clarified by centrifugation at $12,000 \mathrm{rpm}$ at $4{ }^{\circ} \mathrm{C}$ for $15 \mathrm{~min}$. Aliquots of $20 \mu \mathrm{g}$ were run on $7.5 \%$ sodium dodecyl sulfate-polyacrylamide gel electrophoresis (SDS-PAGE), followed by electroblotting onto PVDF membranes (Millipore, Bedford, MA, USA). The ErbB2 protein was detected using Neu anti-ErbB2 mAb (Santa Cruz Biotechnology, Santa Cruz, CA, USA), followed by rabbit anti-mouse horseradish peroxidase-conjugated antibody. The signal intensity of reactive bands was quantitatively measured by a phosphorimager (45-710; Bio-Rad, Hercules, CA, USA).

Enzyme-linked immunosorbent assays (ELISA)

ErbB2-positive gastric tumor cells, harvested in nonenzymatic dissociation solution (Sigma), were washed and transferred to U-bottom microtiter plates $\left(1 \times 10^{5}\right.$ cells per well). After blocking with PBS containing $6 \%$ bovine serum albumin (BSA), cells were treated with the antibodies as previously described [19]. For detection of human antibodies, a peroxidase-conjugated anti-human $\mathrm{IgG}$ (Fc-specific; Sigma) antibody was used. Binding values were determined from the absorbance at $450 \mathrm{~nm}$ and reported as the mean of at least three determinations (standard deviation $\leq 5 \%$ ). 
Cell survival and growth inhibition assays

Gastric tumor cells were seeded in 96-well, flat-bottom plates at a density of $7.5 \times 10^{3}$ well. After addition of the protein under test, viable cells by the trypan blue exclusion test were counted at suitable time intervals. Cell survival was expressed as percent of viable cells in the presence of the protein under test with respect to control cultures grown in the absence of the protein. Typically, cell survival values were obtained from at least three independent experiments in which triplicate counts were determined; standard deviations were less than $5 \%$.

Growth inhibition of gastric tumor cells was also assessed by using the MTS assay (Promega, Madison, WI, USA). This colorimetric method for determining the number of viable cells is based on the bioreduction of 3-(4,5-dimethylthiazol-2-yl)-5-(3-carboxymethoxyphenyl)2-(4-sulfophenyl)-2H-tetrazolium (MTS) by cells to a formazan product that is soluble in tissue culture media and can be detected spectrophotometrically. For this assay, cells were seeded in 96-well plates in complete growth media. After $24 \mathrm{~h}$, cell culture media were replaced with media containing $0.1 \%$ fetal bovine serum (FBS) in the absence or in the presence of Erb-hcAb, which was added at concentrations ranging from 2 to $500 \mathrm{nM}$. At day 1, 2, or 3, 20- $\mu$ l aliquots of MTS (Promega, Madison, WI, USA) were added to each well and plates were incubated at $37{ }^{\circ} \mathrm{C}$ for 2-3 h. The reaction was monitored at A490 nm.

\section{Cell migratory assay}

The migratory behavior of NCI-N87 cells was analyzed in an in vitro wound-healing assay using time-lapse microscopy. Briefly, cells were grown to confluence in the absence or in the presence of Erb-hcAb for $72 \mathrm{~h}$ with culture media containing $0.1 \%$ FBS. Time-lapse microscopy was applied in the last $18 \mathrm{~h}$ of each experiment after applying an artificial wound with a yellow Gilson pipette. Migration was assessed at different time intervals (0-18 h) by measuring the distance in at least six different randomly chosen regions obtained from three independent experiments.

\section{Apoptosis assay}

Cytospin preparations of both floating and attached cells were collected from each sample and fixed with $4 \%$ paraformaldehyde for $15 \mathrm{~min}$ at room temperature. The cells were washed in PBS and permeabilized with $0.1 \%$ Triton X-100 in $0.1 \%$ sodium citrate for 2 min on ice. TdT-mediated dUTP nick-end labeling (TUNEL) analysis was performed by using the In Situ Cell Death Detection kit, fluorescein (Roche) by following the manufacturer's instructions. In each experiment 500 cells were counted, and the standard deviations were calculated on the basis of the results obtained by three independent experiments.

\section{Peripheral blood lymphocytes}

Peripheral blood lymphocytes (PBL) were obtained from peripheral blood mononuclear cells isolated by centrifugation on Lymphoprep gradients (Axis Shield PoC, Oslo, Norway) from normal donor buffy coats obtained from the Blood Bank of the Medical School of the University of Naples 'Federico II.' After the separation, PBL were washed twice and incubated in RPMI 1640 medium (Gibco BRL) for $2 \mathrm{~h}$ at $37{ }^{\circ} \mathrm{C}$ to remove adherent cells. The nonadherent cells were used as natural cytotoxic effectors without any additional treatment.

\section{ADCC tests}

NCI-N87 target cells were detached from culture dishes with a cell dissociation solution (Sigma), transferred to 96-well plates (7,000 cells/well), and incubated for $16 \mathrm{~h}$ at $37{ }^{\circ} \mathrm{C}$. Subsequently, PBMCs from a healthy donor were added at effector:target ratio 10:1 in the absence or presence of increasing concentrations of Erb-hcAb or trastuzumab (5-200 nM) and incubated for 24-48 h at $37{ }^{\circ} \mathrm{C}$. Controls included target cells incubated in the absence of effector cells or in the presence of immunoagents alone. Tumor cell lysis was determined by measuring the release of lactate dehydrogenase (LDH) using an $\mathrm{LDH}$ detection kit (Roche, Mannheim, Germany). Antibody-dependent cellular cytotoxicity was calculated as the percentage of cytolysis measured in the presence of immunoagent and PBL, taking as $100 \%$ the maximal LDH release determined by lysis of target cells with $1 \%$ Triton X-100. Standard deviations (SD) were calculated on the basis of the results obtained from three independent experiments.

In vivo antitumor activity

In vivo experiments were performed with 6-week-old female Balb/c nude mice (Charles River Laboratories, Calco, Italy). NCI-N87 cells $\left(2 \times 10^{6}\right)$ were suspended in $0.2 \mathrm{ml}$ sterile PBS and injected subcutaneously (day 0) in the right paw. At day 10, when tumors were clearly detectable, Erb-hcAb or trastuzumab dissolved in PBS was administered intraperitoneally to two different groups of six mice at doses of $5 \mathrm{mg} / \mathrm{kg}$ body weight, seven times, at 72-h intervals.

Another group of control mice was treated with identical volumes of sterile PBS. During the period of treatment, tumor volumes $(V)$ were measured with a caliper and calculated by the formula of rotational ellipsoid $V=A \times B^{2} /$ 
2 ( $A$ is the axial diameter and $B$ the rotational diameter). SD values were less than $10 \%$. All mice were maintained at the animal facility of the Department of Cellular and Molecular Biology and Pathology, University of Naples Federico II, and received human care in compliance with the guidelines of this institution. The experiments in vivo described herein were conducted in accordance with the Italian regulations for experimentation on animals.

\section{Results}

ErbB2 levels on gastric cancer cells

The level of ErbB2 receptor in gastric tumor cells was investigated by Western blotting analyses of cell extracts from NCI-N87, MKN-7, MKN-45, and AGS tumor cells. Briefly, cells were lysed and analyzed by Western blotting with a commercial anti-ErbB2 antibody (Neu; Santa Cruz Biotechnology) and an anti-actin mAb. As a positive control, we used SKBR3 human breast cancer cells, which express high levels of ErbB2. As shown in Fig. 1a, ErbB2 is significantly expressed in some of the gastric cell lines

a
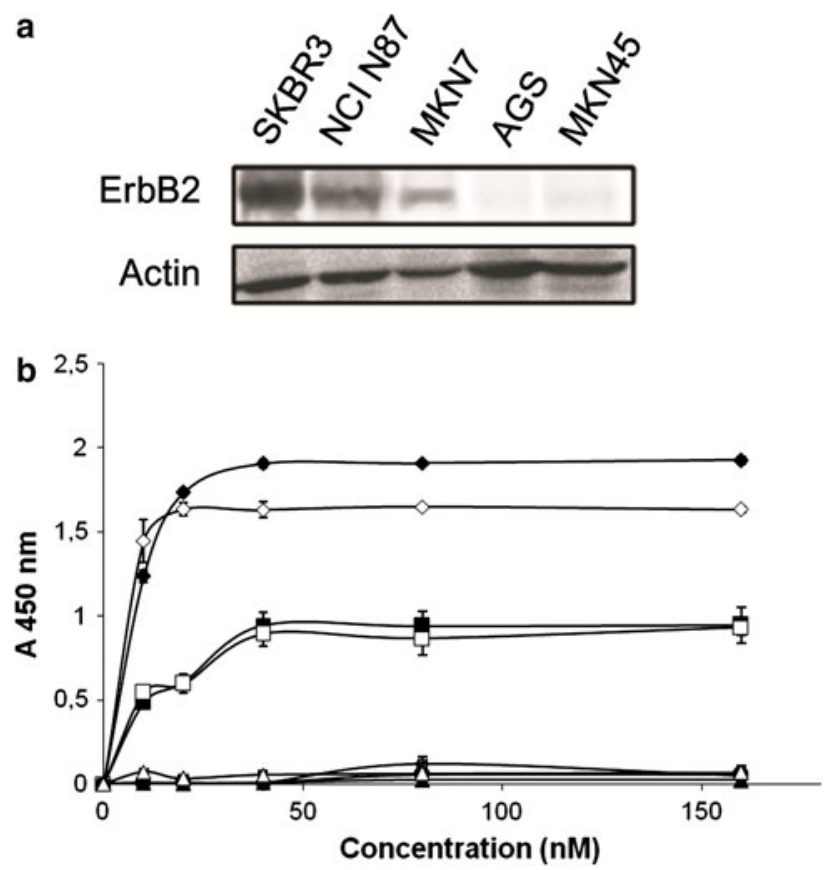

Fig. 1 Levels of ErbB2 and its binding to Erb-hcAb in gastric tumor cells. a ErbB2 expression on human cancer cell lines. Western blotting analyses of ErbB2 protein expression on total cell lysates from NCI-N87, MKN-7, MKN-45, or AGS tumor cells or from mammary SKBR3 cells, used as a positive control. To normalize the band signals, an anti-actin monoclonal antibody (mAb) was used. b Binding curves of the antibodies to gastric cancer cells as determined by ELISA assays. Trastuzumab (empty symbols) or ErbhcAb (black symbols) was tested on NCI-N87 (rhomboids), MKN-7 (squares), MKN-45 (circles), or AGS (triangles) cells tested, such as NCI-N87 and MKN-7, although at lower levels with respect to those observed in mammary carcinoma cells.

These results were confirmed by ELISA assays, performed by incubating the gastric cancer cells with increasing concentrations of Erb-hcAb or trastuzumab. As shown in Fig. 1b, the two antibodies were found to bind to the NCI-N87 and MKN-7 gastric cells tested with high affinities, whereas no significant binding to MKN-45 and AGS cells expressing low levels of ErbB2 was detected.

In vitro effects of Erb-hcAb on gastric cancer cells

The antitumor effects of the antibodies were tested on gastric tumor cells by incubating NCI-N87, MKN-7, MKN-45, and AGS cells in the absence or in the presence of increasing concentrations (50-200 nM) of Erb-hcAb or trastuzumab.

After $72 \mathrm{~h}$, cells were counted and their survival was expressed as percentage of viable cells in the presence of the protein under test, with respect to control cells grown in the absence of the protein. The SD was calculated on the basis of the results obtained from three independent experiments.

As shown in Fig. 2, Erb-hcAb was found to inhibit the growth of ErbB2-positive NCI-N87 and MKN-7 cells tested in a dose-dependent manner, showing a stronger effect with respect to trastuzumab when high protein concentrations were tested. In contrast, no significant effects were observed when Erb-hcAb or trastuzumab was tested on MKN-45 or AGS cells expressing very low levels of ErbB2 (see Fig. 1). These data clearly indicate the specificity of the antiproliferative activity of the anti-ErbB2 antibodies.

Further growth inhibition assays were performed by testing with MTS assay the effects of Erb-hcAb on NCIN87 cells in comparison with those observed on other ErbB2-positive tumor cells from different tissues, such as mammary SKBR3 and MDA-MB-468 or colon SW480 cells, or ErbB2-negative MCF7 cells, used as a control.

As shown in Fig. 3, Erb-hcAb was found to selectively inhibit the proliferation of all the antigen-positive cell lines tested in a dose-dependent manner, with the strongest effect on NCI-N87 gastric tumor cells. When Erb-hcAb was tested on MCF7 control cells, no effects on their proliferation were observed (Fig. 3).

Effects on ErbB2 signaling and determination of the nature of the tumor cell death

We then evaluated by Western blot analysis the effects of treatment with Erb-hcAb on the expression of proteins involved in the ErbB2 pathway in NCI-N87 cells. Erb$\mathrm{hcAb}$ inhibited the phosphorylation of MAPK and Akt 
Fig. 2 In vitro antitumor effects of Erb-hcAb on gastric cancer cells. Dose-response

(rhomboids), MKN-7 (squares), MKN-45 (circles), or AGS

(triangles) cells treated for $72 \mathrm{~h}$ with Erb-hcAb (black symbols) or trastuzumab (empty symbols) curves of NCI-N87

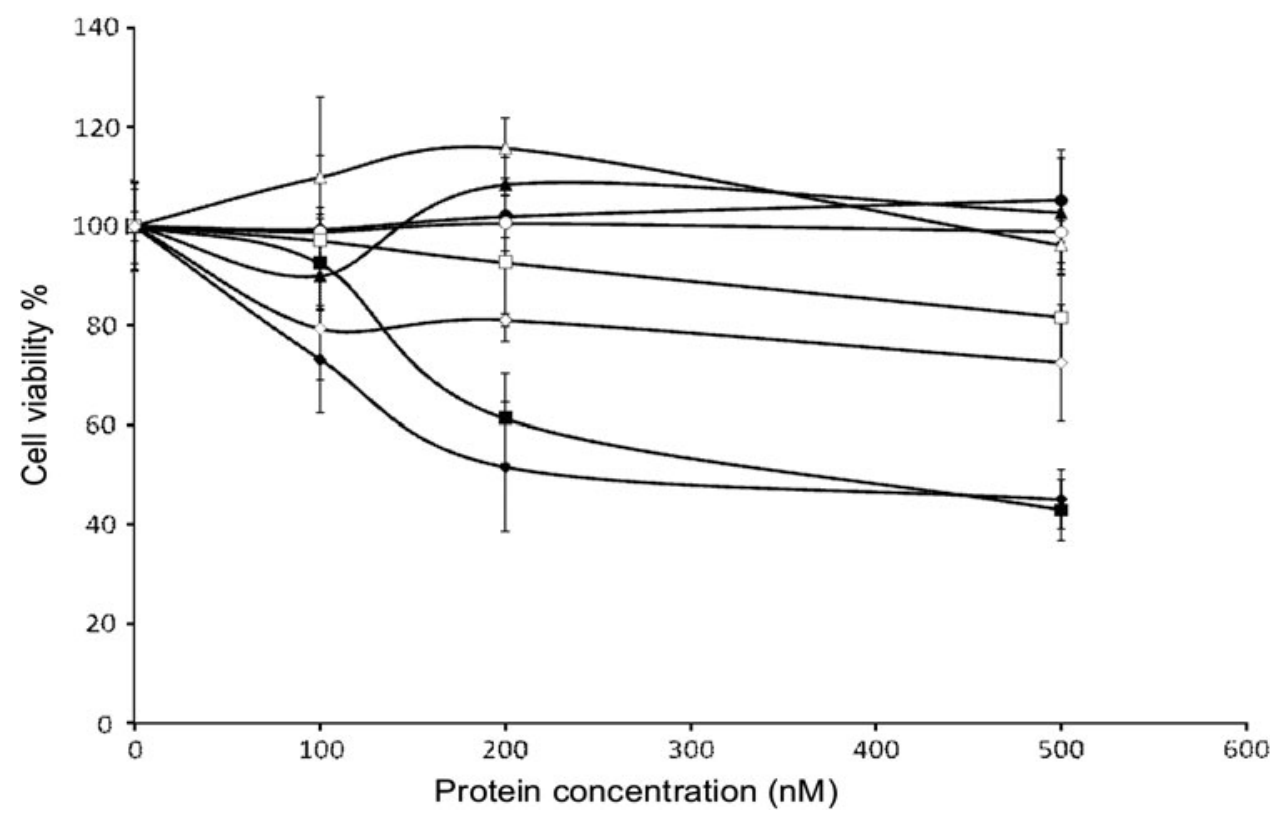

Fig. 3 Dose-response curves for the cell lines indicated in the figure, treated for $72 \mathrm{~h}$ with increasing concentrations of Erb-hcAb

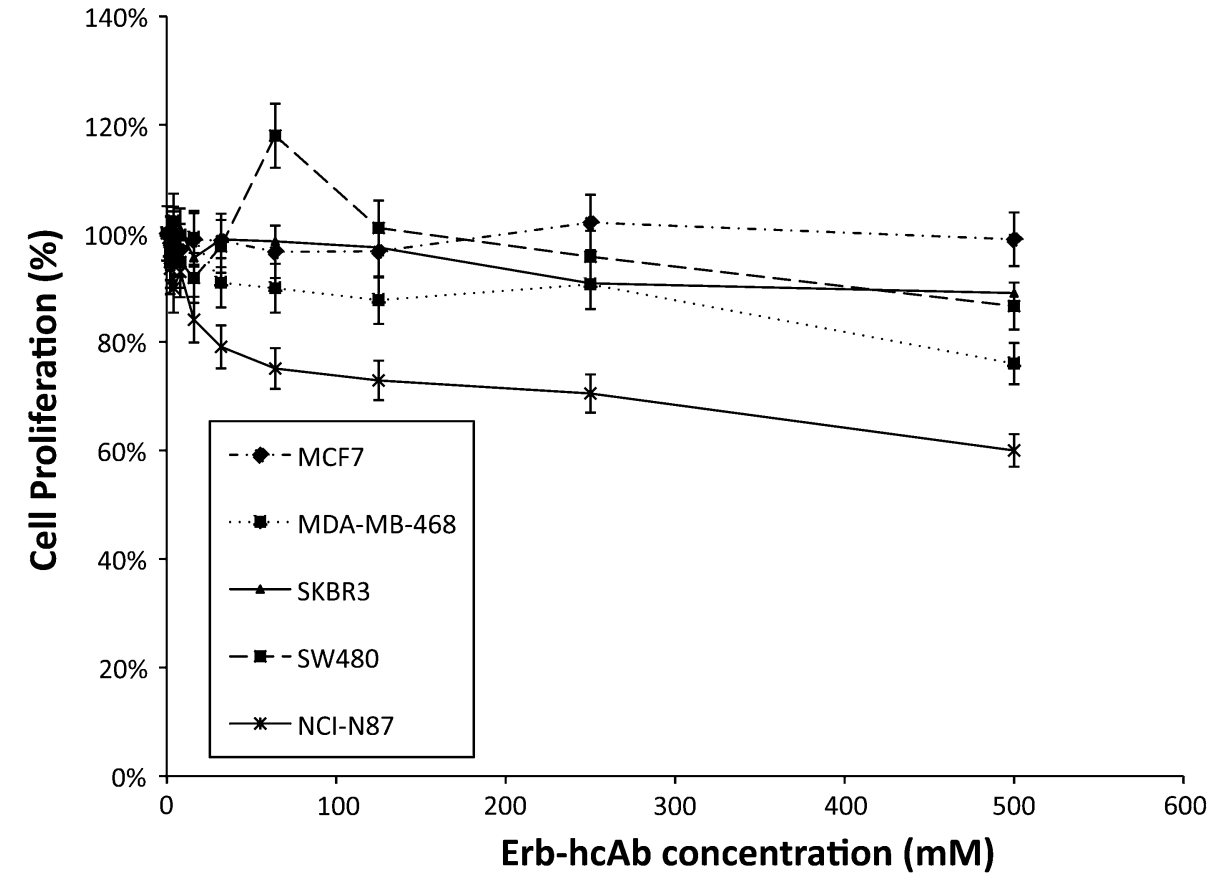

(Fig. 4), thus suggesting that it retains its ability to inhibit the ErbB2 survival pathway in gastric tumor cells.

To determine whether the mechanism of cell death caused by the treatment with Erb-hcAb occurs through induction of apoptosis, we used TUNEL assays. NCI-N87 cells treated with Erb-hcAb for 48 or $72 \mathrm{~h}$ were found to have a 5.5- or 4.5-fold increase in cell death compared to control cells (Fig. 5), respectively.

These results show that the nature of cell death as induced by Erb-hcAb is apoptotic.
Antibody-dependent cellular cytotoxicity (ADCC) as induced by Erb-hcAb

To determine the capacity of Erb-hcAb to trigger ADCC toward antigen-expressing NCI-N87 cells, the target cells were incubated for 24 or $48 \mathrm{~h}$ with effector PBL in the absence or in the presence of increasing concentrations of Erb-hcAb or trastuzumab, used as a positive control. As shown in Fig. 6, Erb-hcAb effectively lysed NCI-N87 target cells in the presence of PBL. The extent of lysis 
reached almost $35 \%$ of treated cells, whereas trastuzumab, used as a positive control, induced about 15-20\% lysis. The basal level of cytotoxicity was measured in the presence of PBL (Fig. 6) or Erb-hcAb alone (data not shown). No effects were detected in parallel assays carried out with

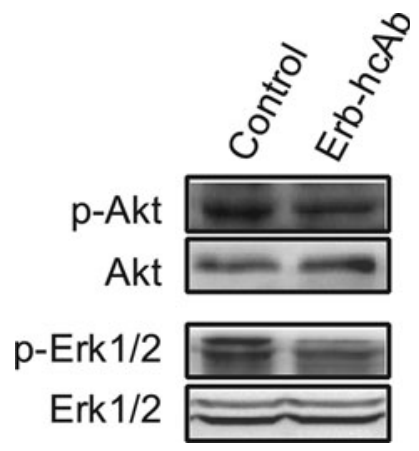

Fig. 4 Effects of Erb-hcAb on signal transduction of ErbB2 in NCIN87 cells. Effects on MAPK and Akt signaling in NCI-N87 tumor gastric cells, as exerted by Erb-hcAb. Reduction in p-Erk1/2 or p-Akt levels was normalized to total Erk2 or Akt, respectively, by phosphor imaging signals detected by Western blotting
ErbB2-negative cells, such as A431 (data not shown), or when Erb-hcAb was replaced by the parental anti-ErbB2 scFv, lacking the Fc domain (data not shown). These results indicate the specificity of the Erb-hcAb-dependent cell-mediated cytolytic activity, clearly based on binding abilities of the immunoagent both to the cognate receptor with its antigen-binding sites and to natural killer cells with its Fc effector domain.

\section{Cell migratory assay}

Cellular motility is a significant process in many biological systems. Most deaths of cancer patients occur not because of the primary tumor but rather from tumor cells that acquire a motile-invasive phenotype and develop metastases.

To test the ability of Erb-hcAb to inhibit the migration of gastric NCI-N87 tumor cells, we carried out woundhealing assays using time-lapse microscopy. Figure 7 shows that NCI-N87 cells treated with Erb-hcAb for $18 \mathrm{~h}$ migrate significantly less than untreated control cells. It
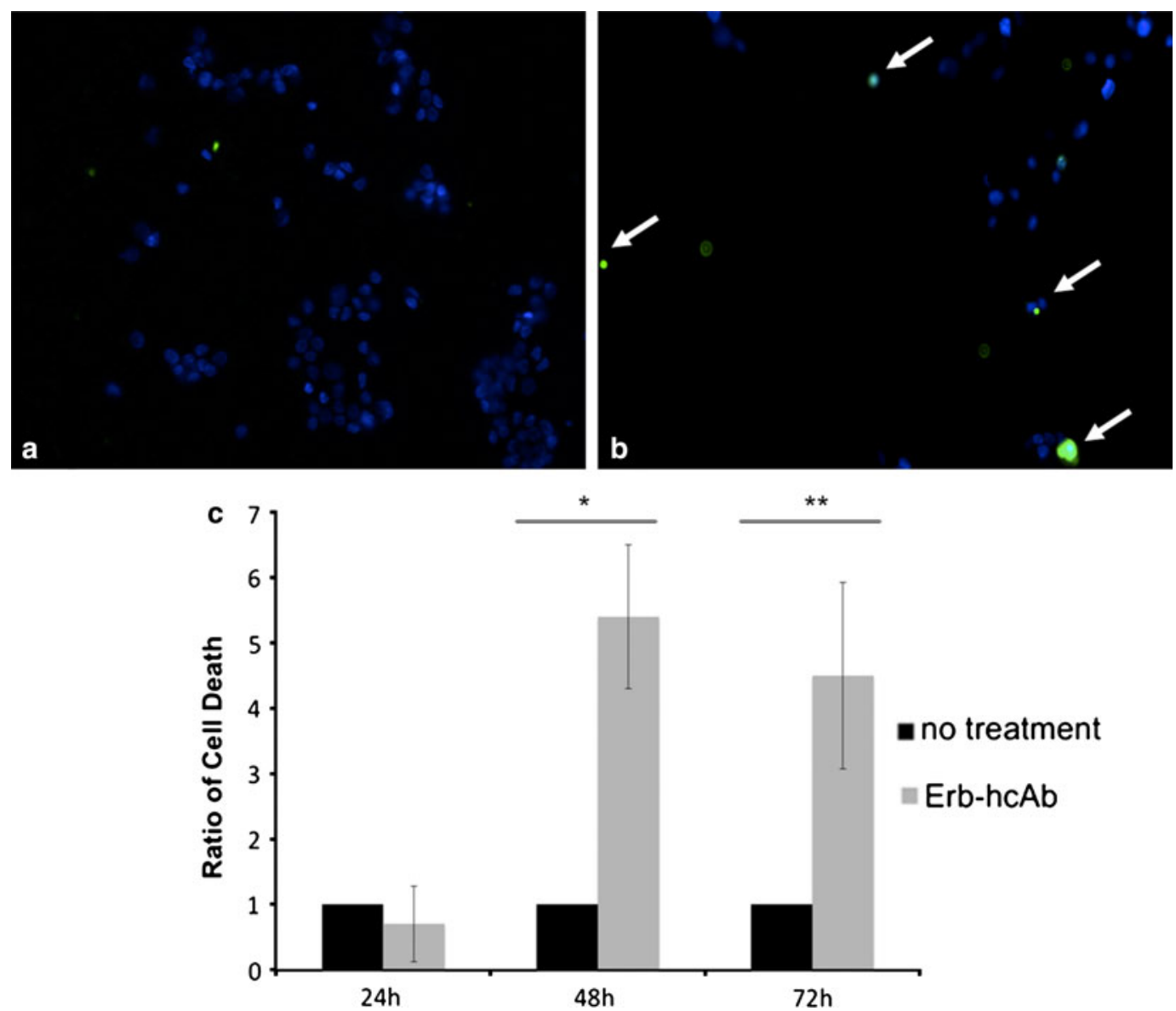

Fig. 5 Induction of apoptosis by Erb-hcAb by TUNEL assay in (a) untreated NCI-N87 cells or (b) cells treated with Erb-hcAb. Arrows indicate dying cells $(\times 200)$. c Ratio of cell death obtained by

comparing untreated control cells and cells treated with Erb-hcAb at different time intervals $(24,48$, and $72 \mathrm{~h})$. Two-tailed Student's $t$ tests were used to calculate the $p$ values. $* p=0.002, * * p=0.01$ 
Fig. 6 Antibody-dependent cytotoxicity assays of ErbhcAb. NCI-N87 cells treated for 24 or $48 \mathrm{~h}$ with peripheral blood lymphocytes (PBL) as effector cells in the absence (straight lines) or in the presence of ErbhcAb (rhomboids) or trastuzumab (squares), respectively
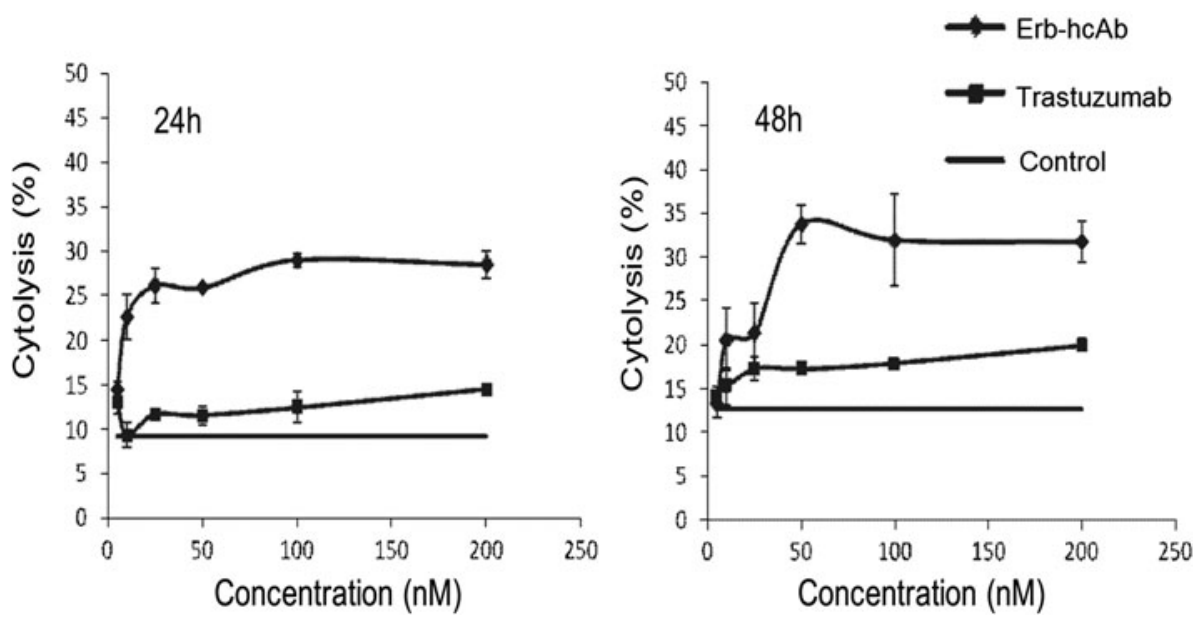

Fig. 7 Wound-healing assay by using time-lapse microscopy after $72 \mathrm{~h}$ treatment in the absence or in the presence of Erb-hcAb. Cell monitoring was conducted in the last $18 \mathrm{~h}$ of the experiment $(\times 400)$. The control cells almost entirely closed the wound (with a $98.9 \%$ closure) whereas the treated cells were only able to close $17.6 \%$ of the wound. The migrated area was quantified by using ImageJ (assessed area represented by yellow outline)

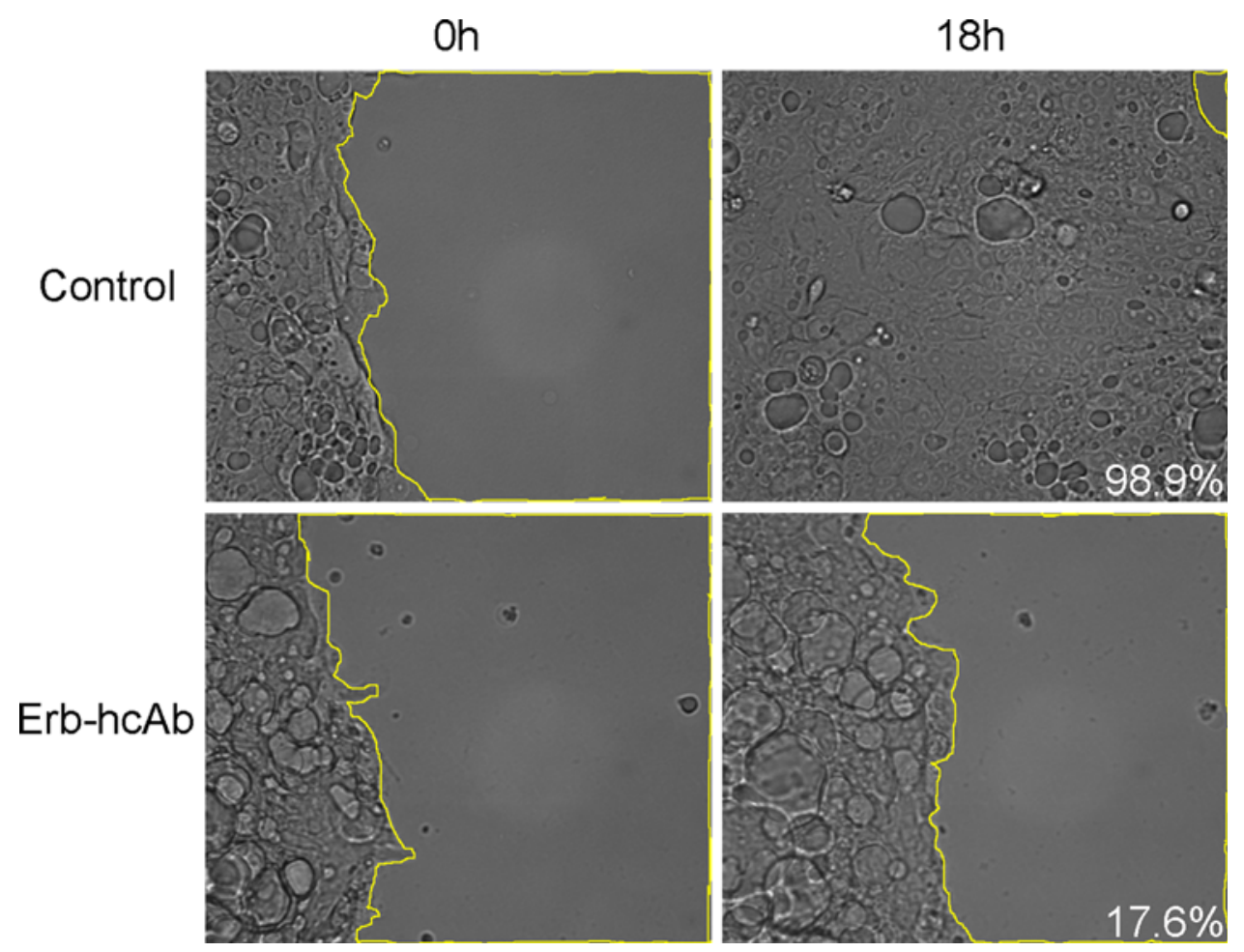

was estimated that control untreated cells almost entirely closed the wound (98.9\%) whereas the Erb-hcAb treated cells were able to cover only $17.6 \%$ of the wound.

In vivo antitumor activity of Erb-hcAb

For in vivo studies, Erb-hcAb was tested on human gastric NCI-N87 cancer cells. The treatment of mice bearing NCI-N87 tumors with seven doses, at 72-h intervals, of $5 \mathrm{mg} / \mathrm{kg}^{-1}$ body wt of Erb-hcAb induced a significant reduction in tumor volume (Fig. 8). In parallel experiments, we tested the effects of trastuzumab, administered as already indicated for Erb-hcAb. Figure 8 shows that trastuzumab, in contrast to Erb-hcAb, showed only slight effects on NCI-N87 gastric tumors. No growth inhibition was observed in mice treated with PBS. During the period of treatment, the animals did not show signs of wasting or other visible signs of toxicity.

\section{Discussion}

The incidence of gastric cancer has been reported to be especially high in Asia, South America, and Eastern Europe [13]. Clinical gastric cancer patients were treated with various types of therapies such as chemotherapy and radiotherapy, although further improvement and progress would be required as the overall survival does not exceed 


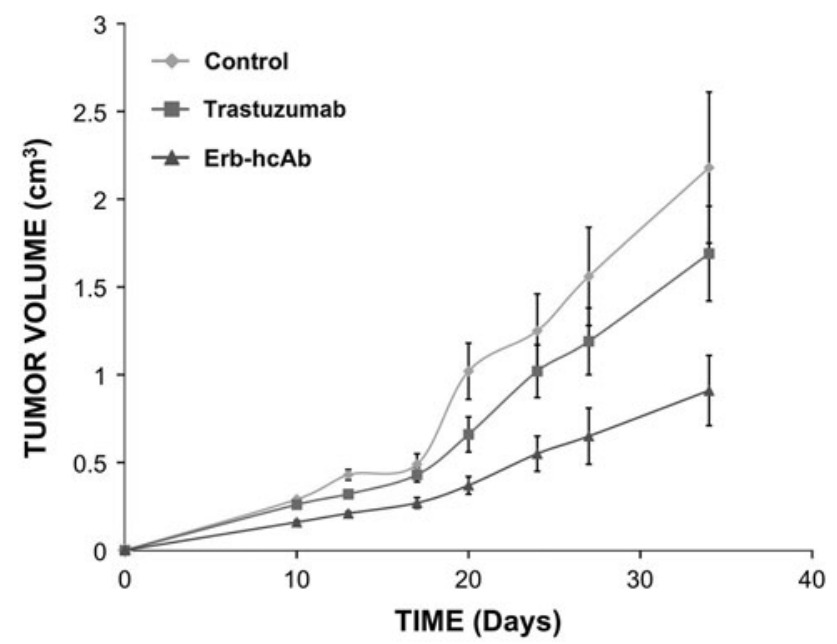

Fig. 8 In vivo antitumor effects of Erb-hcAb on gastric xenografts. Tumor growth was measured in mice inoculated subcutaneously with NCI-N87 cells from a human gastric tumor. Treated mice (triangles) were injected with Erb-hcAb at doses of $5 \mathrm{mg} / \mathrm{kg}$ body weight. Injections were repeated seven times at 72-h intervals. Control mice were treated with sterile PBS solution (rhomboids), or with equimolar doses of trastuzumab (squares)

1 year [27]. Recently, it has been found that upregulation of signaling pathways of the epidermal growth factor receptor family plays a central role in cell differentiation, proliferation, and survival. These findings support the development of antitumor strategies against these targets [27].

The overexpression of ErbB2 protein has been reported in gastric cancer as well as in various other cancers $[4,5]$, even though the frequency data remain controversial [27].

Trastuzumab has been used in combination with chemotherapy in gastric cancer therapy with some success; however, some issues regarding the efficacy, safety, and resistance emerged.

Erb-hcAb is a new human antibody that has been developed to improve the therapy of ErbB2-positive breast cancer because of its convenient molecular size, its activity on trastuzumab-resistant tumor cells $[19,20,26]$, and the lack of cardiotoxicity [23-25].

Recent investigations on the mechanism of antitumor action of this anti-ErbB2 immunoagent have indicated that Erb-hcAb induces $\mathrm{ADCC}$, promotes the homodimerization of ErbB2, which leads to its downregulation and lysosomal degradation, thus inhibiting progression from the $G_{0} / G_{1}$ phase of the cell cycle, and induces apoptosis of ErbB2positive cells [28].

Moreover, Erb-hcAb binds to the soluble extracellular domain of ErbB2 (ErbB2-ECD) with a lower affinity than that for the native receptor inserted in tumor cells. Trastuzumab, by contrast, shows a higher affinity for soluble ErbB2-ECD. Accordingly, ErbB2-ECD abolishes the in vitro antitumor activity of trastuzumab with no effects on the activity of Erb-hcAb [29]. Thus, the fraction of immunoagent neutralized by the free bloodstream extracellular domain is much greater for trastuzumab than for Erb-hcAb, with suggestive effects on therapeutic dosage of the immunoagent.

So far Erb-hcAb has not been extensively studied in preclinical models of gastric cancer. Thus, in the current study, we investigated whether Erb-hcAb would be useful for the treatment of ErbB2-overexpressing gastric cancer by testing its effects either in cell culture or on xenograft models as an indication of its usefulness for treating clinical gastric cancer.

Our in vitro and in vivo data show that Erb-hcAb is effective on gastric cancer cell lines with ErbB2 overexpression, showing an antitumor effect more potent than that observed for trastuzumab. These differences could be explained by the recent interesting finding that Erb-hcAb recognizes an epitope different from that recognized by trastuzumab [29, 30], which has been shown to be responsible for their different effects on signaling in both tumor and cardiac cells [24, 26].

These results therefore confirm the crucial importance of targeting different epitopes of ErbB2 for overcoming resistance and indicate that Erb-hcAb is a new potential tool in the immunotherapy of gastric cancer.

Because trastuzumab has antitumor activity in only one of three of patients, is ineffective on ErbB2 low-expressing tumor cells, and patients often develop resistance to the drug after about 1 year of treatment, attention has been directed toward alternative strategies, such as the use of combinatorial therapeutic protocols. Trastuzumab effectiveness has been found to improve when administered in combination with chemotherapeutic drugs, such as paclitaxel [13] or other anti-ErbB2 antibodies [31]. As for the latter approach, it is necessary that the antibodies used in combination with trastuzumab are directed to epitopes on the target cells different from that recognized by trastuzumab.

We have previously reported that the growth inhibition effects exerted by Erb-hcAb on ErbB2-positive cells are significantly increased when used in combination with either chemotherapeutic drugs or trastuzumab [32]. The effectiveness of taxol and cisplatin was found to increase more than twofold when these drugs were used in combination with Erb-hcAb. Similarly, the cytotoxic action of trastuzumab was enhanced by two- to threefold when ErbB2-positive cells were treated with trastuzumab combined with Erb-hcAb [32].

The results obtained with trastuzumab combined with the erbicin-based immunoagent can be explained by the finding that the epitope recognized by Erb-hcAb is different from that targeted by trastuzumab. 
In conclusion, the results reported here indicate that Erb$\mathrm{hc} \mathrm{Ab}$ has a promising antitumor potential when used in monotherapy, and we can also speculate that it could be favorably used in combination with trastuzumab or conventional chemotherapeutic drugs to achieve synergistic or additive effects on gastric tumor cells, as previously shown for breast tumor cells.

Acknowledgments The authors thank Dr. Philip Cunnah (Biotecnol, S.A., Portugal) for kindly providing the anti-ErbB2 compact antibody Erb-hcAb produced by PER.C6 ${ }^{\circledR}$ cells, Salvatore Sequino for animal care, Dr. G. Terrazzano and Dr. V. Rubino for kindly providing human PBL, and Prof. G. D'Alessio for the critical reading of the manuscript. This study was supported by MIUR (Ministero dell' Istruzione, dell'Università e della Ricerca), and by AIRC (Associazione Italiana per la Ricerca sul Cancro), Italy.

\section{References}

1. Parkin DM, Bray F, Ferlay J, Pisani P. Global cancer statistics, 2002. CA Cancer J Clin. 2005;55(2):74-108.

2. Rivera F, Vega-Villegas ME, Lopez-Brea MF. Chemotherapy of advanced gastric cancer. Cancer Treat Rev. 2007;33(4):315-24.

3. Wesolowski R, Lee C, Kim R. Is there a role for second-line chemotherapy in advanced gastric cancer? Lancet Oncol. 2009;10(9):903-12.

4. Graus-Porta D, Beerli RR, Daly JM, Hynes NE. ErbB-2, the preferred heterodimerization partner of all ErbB receptors, is a mediator of lateral signaling. EMBO J. 1997;16(7):1647-55.

5. Lohrisch C, Piccart M. HER2/neu as a predictive factor in breast cancer. Clin Breast Cancer. 2001;2(2):129-35. discussion 36-37.

6. Fukushige S, Matsubara K, Yoshida M, Sasaki M, Suzuki T, Semba K, et al. Localization of a novel v-erbB-related gene, c-erbB-2, on human chromosome 17 and its amplification in a gastric cancer cell line. Mol Cell Biol. 1986;6(3):955-8.

7. Semba K, Kamata N, Toyoshima K, Yamamoto TA. v-erbBrelated protooncogene, c-erbB-2, is distinct from the c-erbB-1/ epidermal growth factor-receptor gene and is amplified in a human salivary gland adenocarcinoma. Proc Natl Acad Sci USA. 1985;82(19):6497-501.

8. Slamon DJ, Godolphin W, Jones LA, Holt JA, Wong SG, Keith $\mathrm{DE}$, et al. Studies of the HER-2/neu proto-oncogene in human breast and ovarian cancer. Science. 1989;244(4905):707-12.

9. Gravalos C, Jimeno A. HER2 in gastric cancer: a new prognostic factor and a novel therapeutic target. Ann Oncol. 2008;19(9): 1523-9.

10. Tanner M, Hollmen M, Junttila TT, Kapanen AI, Tommola S, Soini Y, et al. Amplification of HER-2 in gastric carcinoma: association with topoisomerase II-alpha gene amplification, intestinal type, poor prognosis and sensitivity to trastuzumab. Ann Oncol. 2005;16(2):273-8.

11. Romond EH, Perez EA, Bryant J, Suman VJ, Geyer CE Jr, Davidson NE, et al. Trastuzumab plus adjuvant chemotherapy for operable HER2-positive breast cancer. N Engl J Med. 2005; 353(16):1673-84.

12. Stebbing J, Copson E, O'Reilly S. Herceptin (trastuzamab) in advanced breast cancer. Cancer Treat Rev. 2000;26(4):287-90.

13. Fujimoto-Ouchi K, Sekiguchi F, Yasuno H, Moriya Y, Mori K, Tanaka Y. Antitumor activity of trastuzumab in combination with chemotherapy in human gastric cancer xenograft models. Cancer Chemother Pharmacol. 2007;59(6):795-805.
14. Kim SY, Kim HP, Kim YJ, Oh do Y, Im SA, Lee D, et al. Trastuzumab inhibits the growth of human gastric cancer cell lines with HER2 amplification synergistically with cisplatin. Int J Oncol. 2008;32(1):89-95.

15. Matsui Y, Inomata $M$, Tojigamori $M$, Sonoda K, Shiraishi N, Kitano S. Suppression of tumor growth in human gastric cancer with HER 2 overexpression by an anti-HER 2 antibody in a murine model. Int J Oncol. 2005;27(3):681-5.

16. Bang YJ, Van Cutsem E, Feyereislova A, Chung HC, Shen L, Sawaki A, et al. Trastuzumab in combination with chemotherapy versus chemotherapy alone for treatment of HER2-positive advanced gastric or gastro-oesophageal junction cancer (ToGA): a phase 3, open-label, randomised controlled trial. Lancet. 2010;376(9742):687-97.

17. Okines AF, Cunningham D. Trastuzumab in gastric cancer. Eur J Cancer. 2010;46(11):1949-59.

18. Nahta R, Yu D, Hung MC, Hortobagyi GN, Esteva FJ. Mechanisms of disease: understanding resistance to HER2-targeted therapy in human breast cancer. Nat Clin Pract Oncol. 2006;3(5): 269-80.

19. De Lorenzo C, Tedesco A, Terrazzano G, Cozzolino R, Laccetti $\mathrm{P}$, Piccoli R, et al. A human, compact, fully functional anti-ErbB2 antibody as a novel antitumour agent. Br J Cancer. 2004;91(6): $1200-4$.

20. De Lorenzo C, Palmer DB, Piccoli R, Ritter MA, D’Alessio G. A new human antitumor immunoreagent specific for ErbB2. Clin Cancer Res. 2002;8(6):1710-9.

21. De Lorenzo C, D'Alessio G. Human anti-ErbB2 immunoagents: immunoRNases and compact antibodies. FEBS J. 2009;276(6): $1527-35$.

22. Chien KR. Myocyte survival pathways and cardiomyopathy: implications for trastuzumab cardiotoxicity. Semin Oncol 2000; 27(6 suppl 11):9-14 (discussion 92-100).

23. Fedele C, Riccio G, Coppola C, Barbieri A, Monti MG, Arra C, et al. Comparison of preclinical cardiotoxic effects of different ErbB2 inhibitors. Breast Cancer Res Treat. 2012;133(2):511-21.

24. Fedele C, Riccio G, Malara AE, D'Alessio G, De Lorenzo C. Mechanisms of cardiotoxicity associated with ErbB2 inhibitors. Breast Cancer Res Treat. 2012;134(2):595-602.

25. Riccio G, Esposito G, Leoncini E, Contu R, Condorelli G, Chiariello M, et al. Cardiotoxic effects, or lack thereof, of antiErbB2 immunoagents. FASEB J. 2009;23(9):3171-8.

26. Gelardi T, Damiano V, Rosa R, Bianco R, Cozzolino R, Tortora G, et al. Two novel human anti-ErbB2 immunoagents are active on trastuzumab-resistant tumours. Br J Cancer. 2010;102(3):513-9.

27. De Vita F, Giuliani F, Silvestris N, Catalano G, Ciardiello F, Orditura M. Human epidermal growth factor receptor 2 (HER2) in gastric cancer: a new therapeutic target. Cancer Treat Rev 2010;36(suppl 3):S11-S15.

28. De Lorenzo C, Cozzolino R, Carpentieri A, Pucci P, Laccetti P, D'Alessio G. Biological properties of a human compact antiErbB2 antibody. Carcinogenesis (Oxf). 2005;26:1890-5.

29. Troise F, Cafaro V, Giancola C, D'Alessio G, De Lorenzo C. Differential binding of human immunoagents and herceptin to the ErbB2 receptor. FEBS J. 2008;275(20):4967-79.

30. Troise F, Monti M, Merlino A, Cozzolino F, Fedele C, Russo Krauss I, et al. A novel ErbB2 epitope targeted by human antitumor immunoagents. FEBS J. 2011;278(7):1156-66.

31. Spector NL, Blackwell KL. Understanding the mechanisms behind trastuzumab therapy for human epidermal growth factor receptor 2-positive breast cancer. J Clin Oncol. 2009;27(34): $5838-47$.

32. De Lorenzo C, Troise F, Cafaro V, D'Alessio G. Combinatorial experimental protocols for erbicin-derived immunoagents and herceptin. Br J Cancer. 2007;97:1354-60. 